\title{
PRELIMINARY TESTS OF A NEW LOW-COST PHOTOGRAMMETRIC SYSTEM
}

\author{
M. Santise ${ }^{1,2, *}$, K. Thoeni ${ }^{1}$, R. Roncella ${ }^{2}$, S. W. Sloan ${ }^{1}$, A. Giacomini ${ }^{1}$ \\ ${ }^{1}$ Centre for Geotechnical Science and Engineering, The University of Newcastle, 2308 Callaghan, Australia - \\ (marina.santise, klaus.thoeni, scott.sloan, anna.giacomini)@newcastle.edu.au \\ ${ }^{2}$ Dept. of Engineering and Architecture, University of Parma, Parco Area delle Scienze, 181/A, 43124 Parma - \\ riccardo.roncella@unipr.it
}

\section{Commission II}

KEY WORDS: Low Cost, Digital Surface Model, Accuracy Assessment, Photogrammetry, Raspberry Pi, 3D Reconstruction

\begin{abstract}
:
This paper presents preliminary tests of a new low-cost photogrammetric system for 4D modelling of large scale areas for civil engineering applications. The system consists of five stand-alone units. Each of the units is composed of a Raspberry Pi 2 Model B (RPi2B) single board computer connected to a PiCamera Module V2 (8 MP) and is powered by a $10 \mathrm{~W}$ solar panel. The acquisition of the images is performed automatically using Python scripts and the OpenCV library. Images are recorded at different times during the day and automatically uploaded onto a FTP server from where they can be accessed for processing. Preliminary tests and outcomes of the system are discussed in detail. The focus is on the performance assessment of the low-cost sensor and the quality evaluation of the digital surface models generated by the low-cost photogrammetric systems in the field under real test conditions. Two different test cases were set up in order to calibrate the low-cost photogrammetric system and to assess its performance. First comparisons with a TLS model show a good agreement.
\end{abstract}

\section{INTRODUCTION}

The availability of cheap credit card sized single board computers such as the Raspberry Pi (RPi) has enabled the creation of numerous automated camera systems and monitoring systems. Such systems have a very low power consumption and provide fast processing ability at low cost. The applicability of RPi has been demonstrated in a variety of problems beyond the educational context, including health supply chains monitoring (Schön et al., 2014), fire alarm system (Bahrudin et al., 2013), smart city applications (Leccese et al., 2014), environmental monitoring (Nikhade, 2015). Additionally, RPi was also used as an embedded sensor control unit for automatic deformation monitoring by Engel and Schweimler (2015). A detailed discussion on its potential use for crowdsourcing applications in climate and atmospheric sciences is provided in Muller et al. (2015). The combination of the RPi with the PiCamera module is very popular in home security applications (Prasad et al., 2014 and Nguyen at al., 2015), in creating depth estimation systems (Zhuang, 2016) in particular for UAV usage (Neves at al., 2013 and Cooper et al., 2017), in traffic monitoring (Kochláň et al., 2014), in agriculture (Jindarat et al., 2015), and also in environmental sciences (Ferdoush et al., 2014).

The new photogrammetric system presented in this paper is intended for civil and mining engineering applications, in particular for monitoring rock cuttings along major roads and railway networks and high vertical rock surfaces within mining operations. The system is an extension of the stereophotogrammetric system proposed by Roncella and Forlani (2015) and developed to detect changes in Digital Surface Models (DSM) with a scheduled frequency.

This study proposes a new low-cost and innovative multi-stereo acquisition system consisting of several stand-alone units. Each unit is composed of a Raspberry Pi 2 Model B (RPi2B) single board computer (Raspberry Pi 2 Model B, 2017) connected to a
PiCamera Module V2 of 8 MP (Raspberry Pi Camera Module V2, 2017). Each unit is powered by a $10 \mathrm{~W}$ solar panel, which makes them very versatile and easy to install. The acquisition of the images is performed automatically using Python scripts and the OpenCV library. Images are recorded at different times during the day and automatically uploaded onto a remotely accessible FTP server for further processing.

The paper presents a series of preliminary tests conducted to assess the performances of the low-cost sensors used in the system and the quality of the generated DSM.

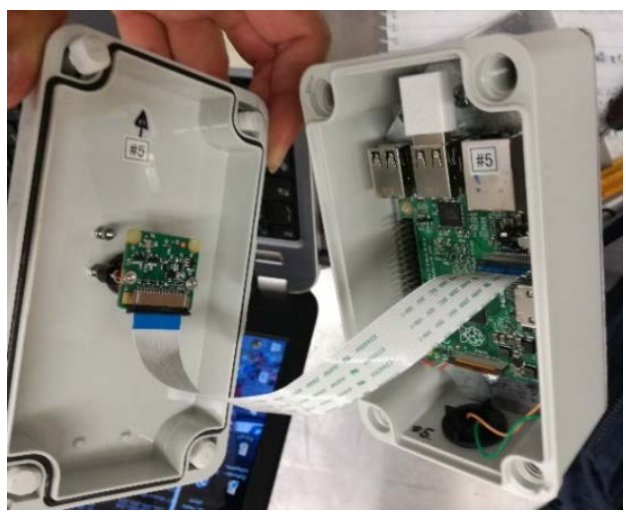

Figure 1. The RPi2B connected with the PiCamera Module V2 in the water proof case designed for the installation of the system in the field.

\section{PHOTOGRAMMETRIC SYSTEM}

A new low-cost photogrammetric system consisting of five stand-alone units is hereby presented. Each unit is composed of an RPi2B single board computer connected to a PiCamera 
Module V2 (Figure 1) and is powered by a $10 \mathrm{~W}$ solar panel that provides continuous recharging to a 16 Ah battery. In case of persistent cloud coverage, the autonomy of the system results in about 5 days.

The RPi2B comes with 4 USB ports, a full HDMI port, an Ethernet port and a slot for a Micro SD card for the operating system installation. Additionally, each RPi2B is equipped with a Wi-Fi dongle which allows communicating with a Wi-Fi network. Hence, the system can be controlled remotely. The camera has a resolution of $8 \mathrm{MP}$, a focal length of $3.04 \mathrm{~mm}$ (angle of view equivalent to that of a $29 \mathrm{~mm}$ lens in $\mathrm{FX}$ format) and a horizontal field of view of $62.2^{\circ}$. For weather and dust resistance purposes, all the major components of the system are enclosed in an IP66 (International Protection Class) weatherproof case (Figure 1). All power connections, camera and battery cases are conceived to be sealed. Power wires are welded to the RPi2B to be connected with the power system through a cable gland. The PiCamera is screwed at the cover case by means of a mounting system. A circular opening has been created on the cover case for the camera. This view hole is kept as small as possible and it is sealed with a circular glass lens.

The free operating system Raspbian is installed on all five RPi2B. The automatic camera acquisition and upload on the FTP server are set up using Python scripts.

For monitoring purposes the units have to be synchronised to simultaneously collect images at the same instant. This can either be achieved using a manual or remote trigger. In our case the cameras are all connected to the same Wi-Fi network, hence, they have approximately the same system time. The image acquisition scheduling is set by the time-based job scheduler software Cron (Cron, 2017).

Considering the low resolution of the sensor and quality of the optics, the assessment of the performance of the Raspberry $\mathrm{Pi}$ camera for photogrammetric purposes is not trivial. In addition, the acquired images can be very noisy in comparison to stable optics and bigger sensor sizes of commonly used digital singlelens reflex cameras.

The photogrammetric system is intended to be installed in mine sites or areas around major infrastructures where distances between cameras and the monitored slopes and objects can be around $100 \mathrm{~m}$. This could, therefore, increase the Ground Sample Distance (GSD) and consequently affect the precision of the monitoring system. The quality of the images could be improved by reducing the noise and the GSD. The latter, however, can only be achieved by changing the focal length and its application was not considered in this work.

The noise reduction is instead addressed in the current study. The OpenCV library is used for achieving an average of multiple captured images. It is assumed that this would reduce the noise. Thus, for this purpose, a preliminary series of tests has been conducted by using the strategy of capturing and averaging the images, followed by the study of the image quality. Additionally, the study investigates the design of the photogrammetric network and the validation of its accuracy.

Finally, the subsequent quality of the photogrammetric digital surface models is assessed. For this purpose, even if the full photogrammetric system consists of 5 units, the current phase of the research applies to the performance's assessment of only one RPi2B (i.e., the same unit is used to capture all the images).

\section{METHODOLOGY}

Two different test sites were considered to calibrate the low-cost photogrammetric system and to assess its performance.

The first test series (test site T1) was performed on a building facade at the University of Newcastle (Figure 2a) (NSW, Australia). Boxes of various sizes were glued to a vertical external wall of the building. Their position was varied within different image acquisition series. The second test site (T2) included the 4D modelling of a small rock wall at Pilkington Street Reserve near the University of Newcastle (Figure 2b). In this case, images were acquired at different times of the day and using two different acquisition techniques: single image acquisition and average acquisition of three consecutive frames. Table 1 summarises the settings for the image acquisitions for both test sites.

\begin{tabular}{cccc}
\hline \multirow{2}{*}{$\begin{array}{c}\text { Test } \\
\text { Site }\end{array}$} & $\begin{array}{c}\text { Image } \\
\text { Block }\end{array}$ & $\begin{array}{c}\text { Image } \\
\text { Acquisition } \\
\text { Stage/Time }\end{array}$ & $\begin{array}{c}\text { Image Acquisition } \\
\text { Method }\end{array}$ \\
\hline \multirow{3}{*}{ T1 } & T1.1 & No boxes & Single image \\
\cline { 2 - 4 } & $\mathrm{T} 1.2$ & With boxes & Single image \\
\cline { 2 - 4 } & $\mathrm{T} 1.3$ & Boxes moved & Single image \\
\hline \multirow{3}{*}{ T2 } & T2.1 & $11: 00$ & Single image \\
\cline { 2 - 4 } & T2.2 & $12: 00$ & Single image \\
\cline { 2 - 4 } & $\mathrm{T} 2.3$ & $13: 00$ & Single image \\
\cline { 2 - 4 } & $\mathrm{T} 2.4$ & $11: 00$ & Average of three images \\
\cline { 2 - 4 } & $\mathrm{T} 2.5$ & $12: 00$ & Average of three images
\end{tabular}

Table 1 - List of test cases for both test sites and relevant information.

During both campaigns, the images were captured from a distance of about $25 \mathrm{~m}$ and with a base-length of about $6 \mathrm{~m}$. Thus, the a priori accuracy study indicates an expected accuracy of about $3.8 \mathrm{~cm}$. The GSD is about $1 \mathrm{~cm} /$ pix.

It is important to notice that the images were acquired assuming a slight convergent geometric configuration, but not in fixed positions. Therefore, the camera poses are slightly different for each image block in $\mathrm{T} 1$ and $\mathrm{T} 2$.

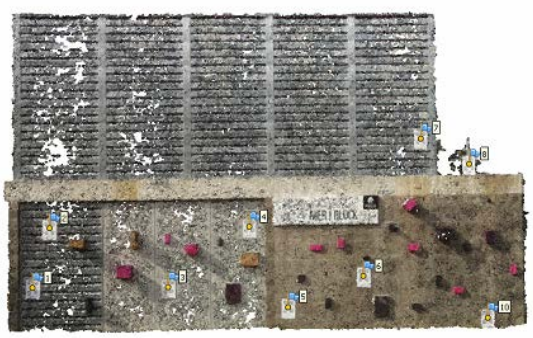

(a)

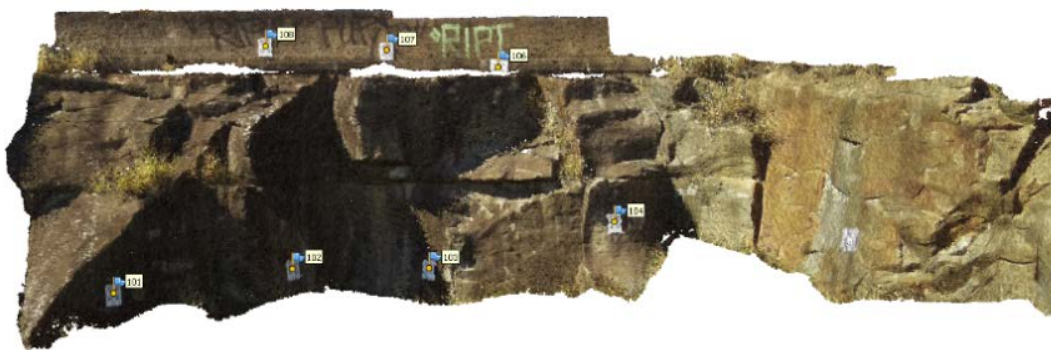

(b)

Figure 2. (a) 3D model of test site T1 with the building covered by targets and boxes of different sizes. (b) 3D model of the rock face of test site $\mathrm{T} 2$ with targets. 
Different calibration techniques can be used for the calibration of the interior orientation (IO) parameters (including lens distortion). A diffuse and dense control point network over the object of interest can support a self-calibration bundle block adjustment (BBA) including automatically extracted tie points. Thus, all the relevant parameters, (interior and exterior orientation (EO) parameters) can be estimated in a single step. This means recovering all the area of interest with many control points. However, the operation is not always feasible especially in remote areas where targets can hardly be installed.

Another possibility is a two-stage calibration, where in a first step the IO parameters are estimated (pre-calibration) and then the EO parameters are computed during the BBA.

In this work, both calibration techniques were explored. The images were oriented in self-calibration using Agisoft PhotoScan version 1.3.0 (Agisoft PhotoScan, 2017), while a set of precalibrated IO parameters was estimated following the procedure in the software Agisoft Lens (Agisoft Lens, 2017).

During both tests, targets were evenly distributed around the area of interest. The coordinates of these targets were measured using a total station. Some of the targets were used as Ground Control Points (GCPs) in the BBA and others as check points for the accuracy assessment of the data.

In addition, a reference model was created using a terrestrial laser scanner (TLS) for T2 only. The absolute distances between the digital models were calculated using CloudCompare (CloudCompare, 2017).

In the following sections, more details on the study of the performance assessment of the low-cost sensor and the evaluation of the quality of the digital surface models generated by the low-cost photogrammetric systems in the field under real test conditions will be provided.

\section{RESULTS}

\subsection{Test site $\mathrm{T} 1$}

The first series of tests was performed on a building at the University of Newcastle (see Figure 2a), called test site T1. A wall about $5 \mathrm{~m}$ high and $11.2 \mathrm{~m}$ long was considered. Nine targets were uniformly distributed on the wall. Twenty-six cardboard boxes of various geometries were glued to the wall to evaluate the minimum traceable displacement. The minimum size of the boxes was $4 \mathrm{~cm}$, which approximately corresponds to the expected accuracy at the distance of $25 \mathrm{~m}$. Boxes of maximum $41 \mathrm{~cm}$ of edge size were considered.

The images were acquired via WI-FI connection by shooting at the wall using different configurations. As reported in Table 1, three image blocks were analysed: i) with only targets on the wall and no boxes (T1.1), ii) with targets and boxes (T1.2), and iii) with targets and some boxes moved to a different position on the wall (T1.3).

A preliminary study on the accuracy of the control points was performed accounting for the IO parameters of the camera in selfcalibration and using also a set of pre-calibrated IO parameters. The study indicated that including additional parameters, such as the coefficient of skew b2 in the self-calibration process, the accuracy on the control points generally decreases, even if the differences compared to the use of IO parameters only do not result very significant (see Table 2 ). In addition, a set of precalibrated IO parameters was used in the BBA. As shown in Table 2, the residuals on the check points decrease for T1.2, while they increase significantly for T1.1 (the residuals double) and for T1.3. As far as the estimated principal distance is considered, (which is probably the parameter whose variation can be most easily interpreted) it is worth noting that the focal lengths estimated in self-calibration for T1.1 and T1.3 (2.8811 and $2.8816 \mathrm{~mm}$ respectively) are quite similar to the value of focal length estimated in the pre-calibration procedure (2.8788 $\mathrm{mm}$ ). Instead, the focal length of T1.2 is 14.7 micron bigger than the focal length estimated in the pre-calibration.

\begin{tabular}{lccc}
\hline $\begin{array}{l}\text { Camera } \\
\text { calibration }\end{array}$ & $\begin{array}{c}\text { T1.1 } \\
\text { RMSE } \\
{[\mathrm{cm}]}\end{array}$ & $\begin{array}{c}\text { T1.2 } \\
\text { RMSE } \\
{[\mathrm{cm}]}\end{array}$ & $\begin{array}{c}\text { T1.3 } \\
\text { RMSE } \\
{[\mathrm{cm}]}\end{array}$ \\
\hline Self-calibration & 0.64 & 0.93 & 0.75 \\
\hline Self-calibration(+b2) & 0.65 & 1.02 & 0.77 \\
\hline Pre-calibration & 1.20 & 0.67 & 1.08 \\
\hline
\end{tabular}

Table 2 - Statistics of the differences on the check points using different BBA for image blocks T1.1, T1.2 and T1.3: in selfcalibration and with a set of pre-calibrated interiors parameters.

Considering the environmental conditions (e.g. the temperature, can influence the focal length) and possible mechanical issues (i.e. the stability of the optics), the self-calibration BBA was chosen as the most appropriate calibration technique for the tests. Thus, the camera calibration parameters estimated in selfcalibration were the principal distance $\mathrm{f}$, the principal point $\left(\mathrm{C}_{\mathrm{x}}\right.$, $\mathrm{Cy})$, and the radial $(\mathrm{K} 1, \mathrm{~K} 2, \mathrm{~K} 3)$ and the tangential (P1, P2) distortion coefficients.

Results obtained in the first set of tests showed that the implemented calibration procedure is still an open issue for this type of low-cost sensors. On one hand, a pre-calibration strategy should grant a more reliable procedure, implementing a properly configured image block geometry and reducing unwanted and potentially detrimental correlations between parameters. On the other hand, the results showed that using a fixed set of calibration parameters could produce inaccurate results, most probably due to the unstable geometry of the optics and acquisition sensor. In this context, a self-calibration procedure can significantly improve the final output. However, considering that just few (in the worst case just two) acquisition units are used (each one with its own calibration parameters) and probably just few (or none) ground control points are available to constrain the BBA, it is very likely that self-calibration could results in an ill-posed estimation of the IO parameters.

Each block was oriented using four of the nine available control points located at the edges of the wall as GCPs. The root mean squares error (RMSE) differences on the check points revealed accuracies below $1 \mathrm{~cm}$ for all test cases as reported in Table 3 .

\begin{tabular}{lcccc}
\hline \multicolumn{5}{c}{ Residuals on the check points } \\
\hline Block & DX [cm] & DY $[\mathrm{cm}]$ & DZ $[\mathrm{cm}]$ & Total $[\mathrm{cm}]$ \\
\hline T1.1 & 0.24 & 0.54 & 0.24 & 0.64 \\
\hline T1.2 & 0.61 & 0.64 & 0.31 & 0.93 \\
\hline T1.3 & 0.19 & 0.60 & 0.41 & 0.75 \\
\hline
\end{tabular}

Table 3 - Statistics of the residuals in $\mathrm{X}, \mathrm{Y}$ and $\mathrm{Z}$ coordinates

on the check points for the photogrammetric blocks of T1.

Then, digital models were generated with the maximum level of details available in PhotoScan. Nevertheless, due to the texture of the wall, the uniform colour patterns, the presence of an opaque surface (on the left), and a uniform metal structure at the left and on the top, the reconstructed models were very noisy. This can also clearly be seen in the Figure 3 which shows the absolute difference between the 3D models of block T1.1 and T1.2. The colour map of the distances displays a few red areas that represent the boxes, and other red areas that correspond to the noise of the models.

Figure 4 shows the comparison between model T1.1 and T1.3. A different colour scale is used and the maximum displacement (red colour) is set to $25 \mathrm{~cm}$. The effect of the noise is reduced (or hidden) and some of the boxes which have been moved in 
between the acquisitions are now visible. The colour map also indicates that the noise is in the order of about $10 \mathrm{~cm}$. Considering the achieved accuracies on the check points and the quality of the digital models, the geometry of the photogrammetric system was well defined, even if the texture of the specific object resulted to be a weak point for the complete reconstruction of the digital models.

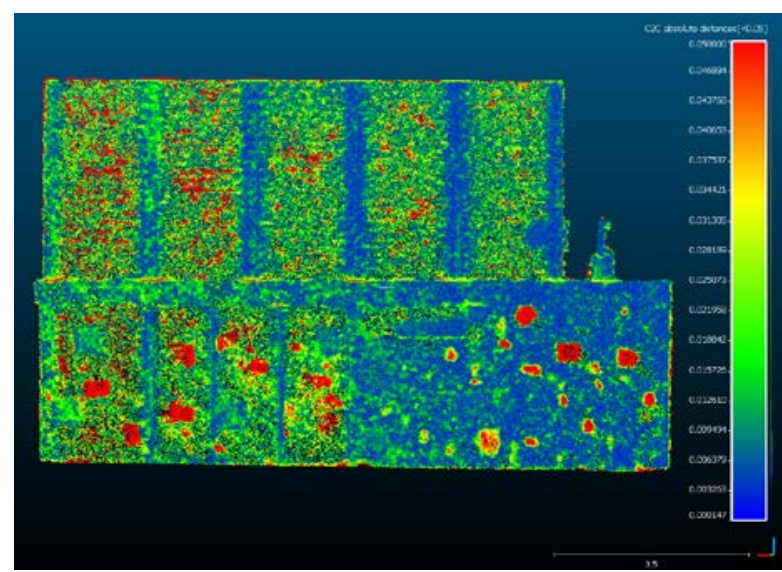

Figure 3. Colour map of distances between the photogrammetric models of T1.1 and T1.2 at the absolute maximum distance of $5 \mathrm{~cm}$.

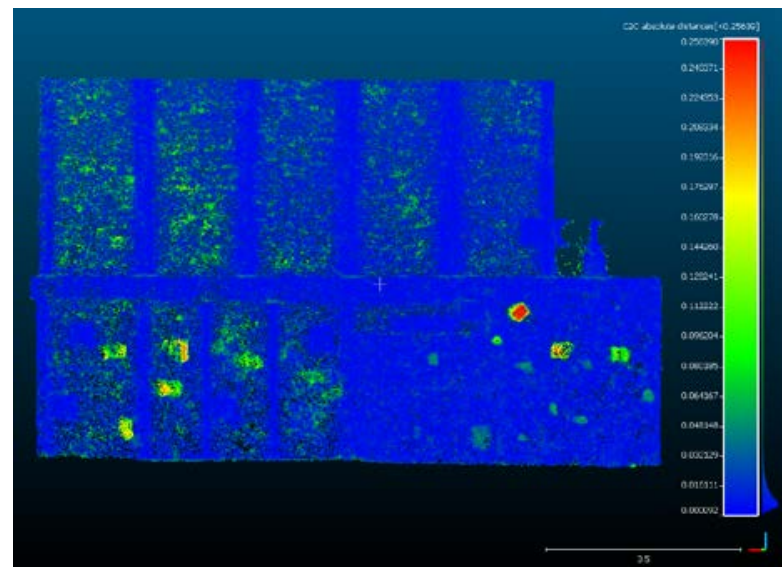

Figure 4. Colour map of distances between the photogrammetric models of T1.1 and T1.3 at the absolute maximum distance of $25 \mathrm{~cm}$.

\subsection{Test site $\mathrm{T} 2$}

Test T2 was realised on a rock wall at the Pilkington Reserve (NSW) (Figure 2b). The tests were conducted with the aim of analysing the quality of the low cost sensor imagery. The rock wall surveyed by means of the PiCamera Module V2 and by the TLS was about $6 \mathrm{~m}$ high and $20 \mathrm{~m}$ long. Targets were located all over the rock surface and their coordinates were measured using a total station.

Two different capturing methods coded by scripts were used: the acquisition of one image or the average of three consecutive frames captured in a specific position (using the OpenCV Libraries). Furthermore, the acquisition was scheduled at different times during the day $(11: 00,12: 00$ and 13:00) to evaluate the influence of the rock wall orientation in respect to the sun exposition during the day on the 3D models reconstruction. The imagery was collected via manual trigger using the Ethernet cable since no Wi-Fi connection was available on site.

Five image blocks were acquired combining different acquisition techniques at different time on a sunny and windy day (see Table 1). Unfortunately, because of strong winds, the average of three images acquisition technique at 13:00 was not possible. Moreover, the residuals on the check points of T2.3 were not obtainable because the wind ripped off most of the targets during that acquisition.

The image block geometry of $\mathrm{T} 2$ was very similar to the one used in T1. The five images were acquired in a slight convergent geometric configuration at the distance from the object of about $23 \mathrm{~m}$ and with a base-length of $4 \mathrm{~m}$. The GSD was $8.6 \mathrm{~mm} /$ pix. The calibration parameters estimated in self-calibration were the principal distance, the principal point, and the radial and the tangential distortion coefficients (Duane, 1971).

Before processing, the images of each block had been masked. Similarly to the procedure followed to process the data of test site $\mathrm{T} 1$, some targets were used as GCPs and others as check points.

\begin{tabular}{lcc}
\hline Block & $\begin{array}{c}\text { RMS reprojection } \\
\text { error [pix] }\end{array}$ & $\begin{array}{c}\text { Estimated principal } \\
\text { distance [mm] }\end{array}$ \\
\hline T2.1 & 0.302 & 2.8633 \\
\hline T2.2 & 0.277 & 2.8819 \\
\hline T2.3 & 0.297 & 2.8902 \\
\hline T2.4 & 0.333 & 2.8520 \\
\hline T2.5 & 0.31 & 2.8688 \\
\hline
\end{tabular}

Table 4 - Photogrammetric blocks specifications of T2: the RMS reprojection error in pixel and the estimated principal distance in $\mathrm{mm}$.

Table 4 reports the RMS of the reprojection errors for each block. The mean value is about 0.30 pixel. In particular, bigger RMS values are observed for the blocks acquired using the average of three images acquisition technique. The estimated principal distance after self-calibration is also reported in Table 4.

\begin{tabular}{lcccc}
\hline \multicolumn{5}{c}{ Residuals on the check points } \\
\hline Block & DX [cm] & DY $[\mathrm{cm}]$ & DZ $[\mathrm{cm}]$ & Total $[\mathrm{cm}]$ \\
\hline T2.1 & 0.51 & 0.68 & 0.57 & 1.02 \\
\hline T2.2 & 0.53 & 0.27 & 0.74 & 0.95 \\
\hline T2.4 & 0.31 & 0.78 & 0.79 & 1.15 \\
\hline T2.5 & 0.57 & 1.10 & 0.56 & 1.36 \\
\hline
\end{tabular}

Table 5 - Statistics of the residuals in $\mathrm{X}, \mathrm{Y}$ and $\mathrm{Z}$ coordinates

on the check points for the photogrammetric blocks of $\mathrm{T} 2$.

The check points indicate a RMSE of just under $1 \mathrm{~cm}$ for all coordinates (Table 5), with the only exception for the $\mathrm{Y}$ coordinate of the block T2.5. Nevertheless, the residuals are definitely in the order of the GSD.

The digital models were also generated at the highest level of details in PhotoScan and compared to the TLS model in CloudCompare. The problem of an inaccurate and noisy 3D reconstruction, as occurred in T1, was avoided by the welldefined texture of the rock face. All photogrammetric point clouds look quite complete and no obvious noise was identified. For example, in Figure 5, one of the photogrammetric digital model is shown with the features of major interest highlighted by circles: the areas with vegetation are indicated with red circles while the shadowed areas with yellow circles.

The five photogrammetric point clouds were registered to the TLS models using the same points located at the boundaries of the wall. Then the absolute distances between the point clouds were calculated using the Cloud-to-Cloud Distance tool with a maximum distance of $5 \mathrm{~cm}$. 


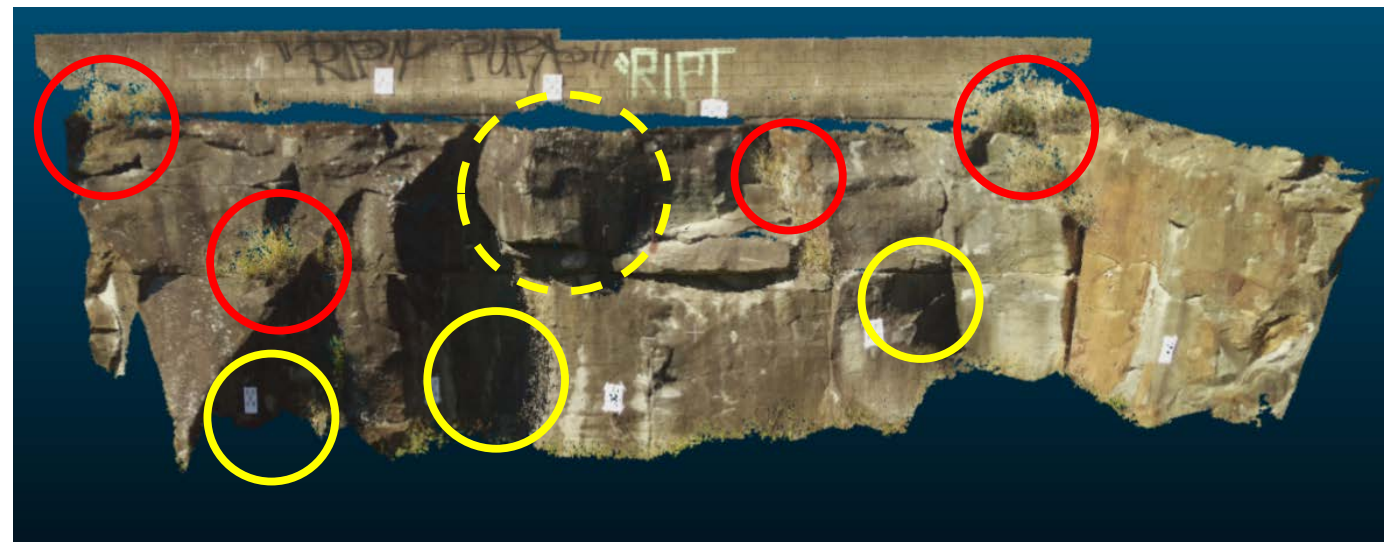

Figure 5. One of the photogrammetric digital models with the areas with vegetation highlighted by red circles and the shadowed areas highlighted by yellow circles: the dashed yellow circle has been further analysed.

The statistics of the absolute differences between the TLS model and the photogrammetric models indicate mean values of about $1.3 \mathrm{~cm}$ with a standard deviation of $0.9 \mathrm{~cm}$ (see Table 6). This latter is again in the order of the GSD. The highest values of the RMS are recorded for T2.5, as it happened for the greater residuals on the check points after the blocks orientation.

The mean values indicates the presence of a systematic effect for all the models, probably due to the estimated interior orientation parameter in the self-calibrating BBA.

\begin{tabular}{lccccc}
\hline \multicolumn{6}{c}{ Absolute distances TLS - Photogrammetric models } \\
\hline Block & T2.1 & T2.2 & T2.3 & T2.4 & T2.5 \\
\hline Mean [m] & 0.012 & 0.013 & 0.012 & 0.013 & 0.014 \\
\hline St. Dev. [m] & 0.008 & 0.009 & 0.008 & 0.010 & 0.010 \\
\hline RMSE [m] & 0.015 & 0.016 & 0.014 & 0.016 & 0.017 \\
\hline
\end{tabular}

Table 6 - Absolute distances between the TLS and the photogrammetric models.

Looking at the standard deviation of the residuals, which should correspond to the noise of the photogrammetric models with respect to the reference models, it can be seen that the models generated with the single image acquisition method are more accurate than the models generated with the average of three image acquisition method.

Likely, during the acquisition of three images, the camera could have been affected to trembling by the strong wind as well as the pictured scene (e.g. the vegetation). Therefore, in this specific case, averaging the images in a time-lapse of 1 second did not improve the quality of the photos.

Figure 6 and Figure 7, for example, show the colour maps of the absolute distance differences in the range of $5 \mathrm{~cm}$ between the TLS model and the photogrammetric digital model of T2.1 and T2.5 respectively. In particular, major differences with the ground truth model are observed in areas with vegetation and shadow. Thus, taking this aspect into account, the vegetation was also affected by the strong wind and, therefore, another comparison excluding these areas was carried out (red circles in Figure 5).

As reported in Table 7, the standard deviation of the absolute differences is of the same order for all the point clouds, included the models generated with the average of three image acquisition method. In addition, the average mean value is reduced, but evident systematic effects can still be noted.

In order to quantify the noise of the models, other comparisons were made between photogrammetric models, excluding the vegetation areas.

\begin{tabular}{lccccc}
\hline \multicolumn{6}{c}{ Absolute distances TLS - Photogrammetric models } \\
No vegetation areas \\
\hline Block & T2.1 & T2.2 & T2.3 & T2.4 & T2.5 \\
\hline Mean [m] & 0.011 & 0.013 & 0.010 & 0.012 & 0.013 \\
\hline St. Dev. [m] & 0.008 & 0.009 & 0.006 & 0.008 & 0.009 \\
\hline RMSE [m] & 0.014 & 0.015 & 0.012 & 0.014 & 0.016
\end{tabular}

Table 7 - Absolute distances between the TLS and the photogrammetric models without areas of vegetation.

In particular, Table 8 summarises the absolute differences between the photogrammetric models acquired with the same capturing method at different time.

\begin{tabular}{|c|c|c|c|}
\hline \multicolumn{4}{|c|}{$\begin{array}{l}\text { Absolute distances between photogrammetric models } \\
\text { No vegetation areas }\end{array}$} \\
\hline Method & \multicolumn{2}{|c|}{ Single image } & $\begin{array}{l}\text { Average of } \\
\text { three images }\end{array}$ \\
\hline Comparison & $\mathrm{T} 2.1-\mathrm{T} 2.2$ & $\mathrm{~T} 2.1-\mathrm{T} 2.3$ & T2.4 - T2.5 \\
\hline Mean [m] & 0.013 & 0.011 & 0.012 \\
\hline St. Dev. [m] & 0.009 & 0.007 & 0.009 \\
\hline RMSE [m] & 0.016 & 0.013 & 0.015 \\
\hline
\end{tabular}

Table 8 - Statistics of absolute distances between the photogrammetric models using the same capture mode at

different times without vegetation areas: the first two differences are related to the models obtained by single image capture mode, the last difference is related to the block acquired using the average of three images.

Table 9 shows the absolute differences between the photogrammetric block acquired at the same time of the day with different capturing method.

\begin{tabular}{lcc}
\hline \multicolumn{3}{c}{ Absolute distances between photogrammetric models } \\
No vegetation areas \\
\hline Time & $11: 00$ & $12: 00$ \\
\hline Comparison & $\mathrm{T} 2.1-\mathrm{T} 2.4$ & $\mathrm{~T} 2.2-\mathrm{T} 2.5$ \\
\hline Mean [m] & 0.009 & 0.012 \\
\hline St. Dev. [m] & 0.007 & 0.009 \\
\hline RMSE [m] & 0.012 & 0.015 \\
\hline
\end{tabular}

Table 9 - Statistics of absolute distances between the photogrammetric models at the same time of the day with different capturing mode without vegetation areas. 


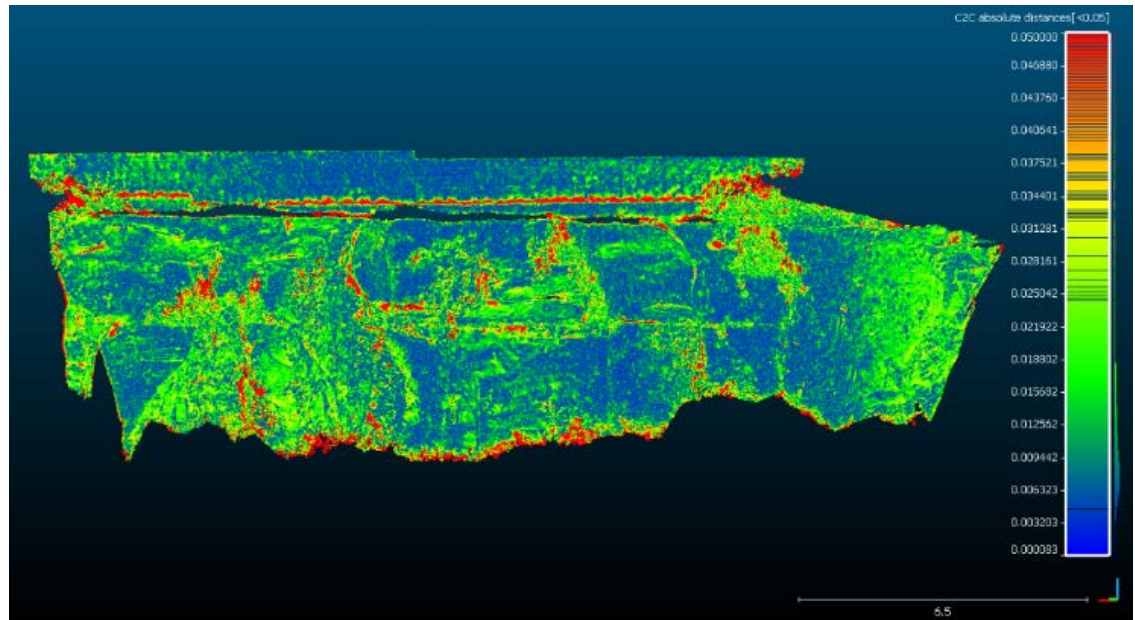

Figure 6. Colour map of absolute distance differences between the TLS model and the block T2.1 photogrammetric digital model in the range of $5 \mathrm{~cm}$ for $\mathrm{T} 2$.

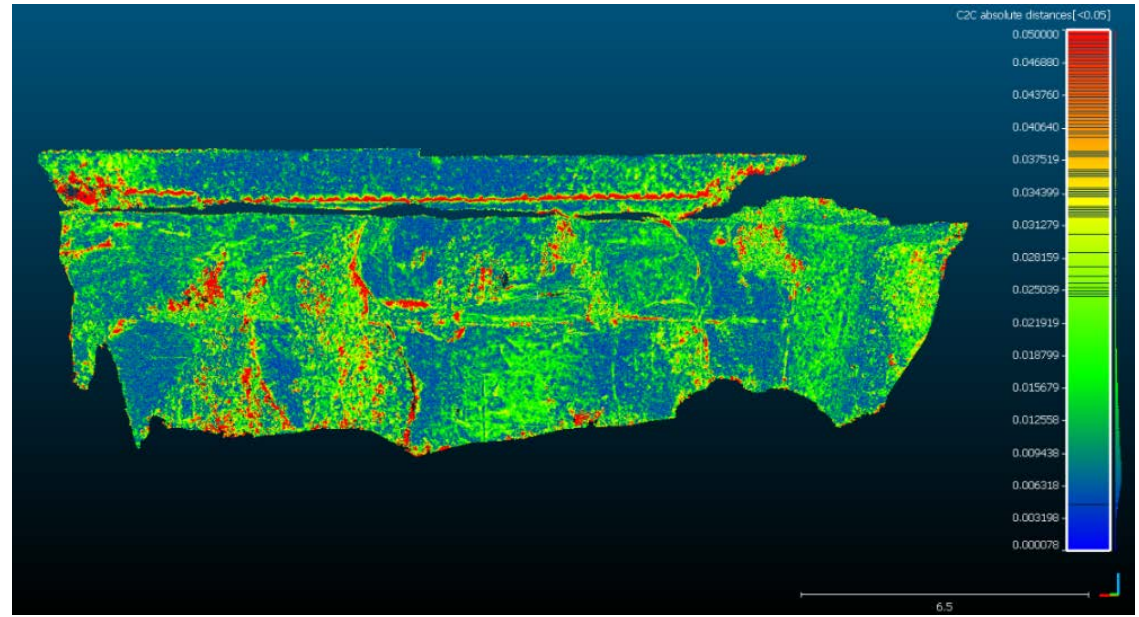

Figure 7. Colour map of absolute distance differences between the TLS model and the block T2.5 photogrammetric digital model in the range of $5 \mathrm{~cm}$ for $\mathrm{T} 2$.

The major residuals are measured by comparing different times of acquisition as different shadows could arise according to the sunlight orientation.

The influence of the orientation of the sun is highlighted by the comparison shown at the top of Figure 8, where the detail of a unique rock feature is depicted for blocks T2.1, T2.2 and T2.3.

Especially for image block T2.2, where the shadowed area is more extended, the comparison of the photogrammetric model with the TLS model shows major differences as visible in the bottom of Figure 8. All the distances colour map are displayed with a colour scale of $5 \mathrm{~cm}$. The comparison of image block T2.3, captured in better exposure condition, reveals greater uniformity and consistency with the TLS model.

Looking at Table 10, the effect is confirmed by the statistical analysis of the absolute distances between the TLS and the photogrammetric models: model T2.3 results the most consistent with the TLS measurements, while model T2.2 the less accurate. This highlights the significance of the lighting conditions during image acquisition, especially for such low-cost sensors.

At this stage, the comparison between the two different acquisition methods shows that averaging multiple images does not represent a significant advantage. In addition, analyses conducted at test site T2 reveal that the PiCamera Module V2 produces images that are suitable for the complete reconstruction of the digital models. In fact, in the worst case, the noise is twice the GSD but always under the expected accuracy.

\begin{tabular}{lccc}
\hline \multicolumn{4}{c}{ Absolute distances TLS - Photogrammetric models } \\
Rock feature \\
\hline Method & \multicolumn{3}{c}{ Single image } \\
\hline Block & T2.1 & T2.2 & T2.3 \\
\hline Mean [m] & 0.013 & 0.015 & 0.011 \\
\hline St. Dev. [m] & 0.009 & 0.011 & 0.008 \\
\hline RMSE [m] & 0.016 & 0.018 & 0.014 \\
\hline
\end{tabular}

Table 10 - Absolute distances between the TLS and the photogrammetric models generated with the single image acquisition method on a detail of a unique rock feature for T2.1, T2.2 and T2.3 blocks. 

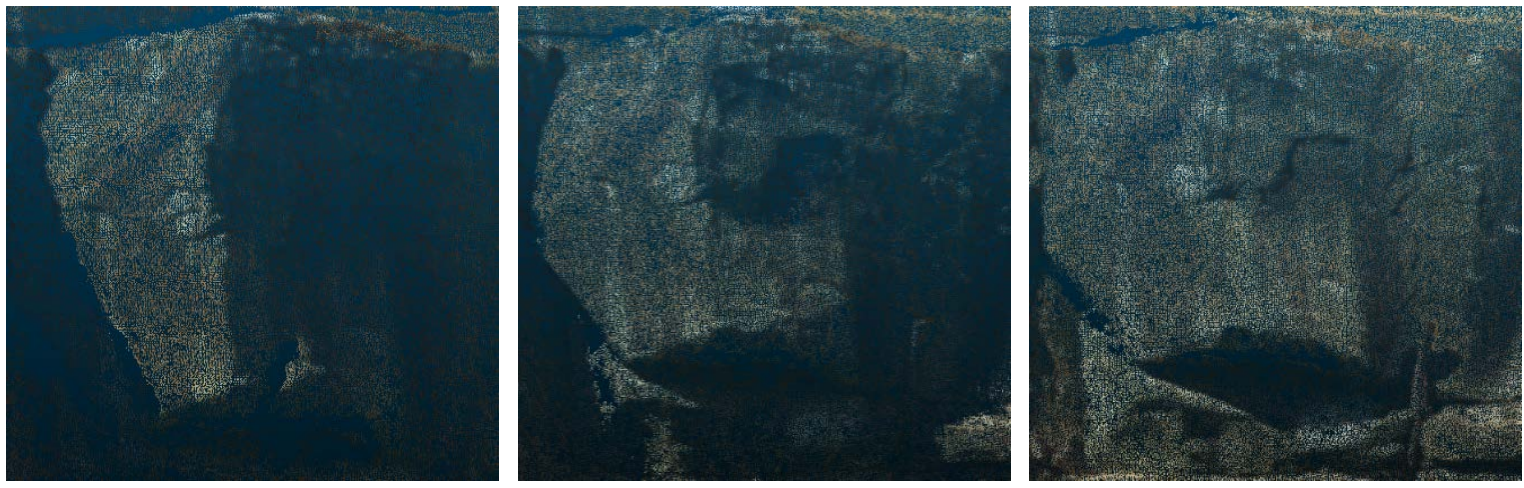

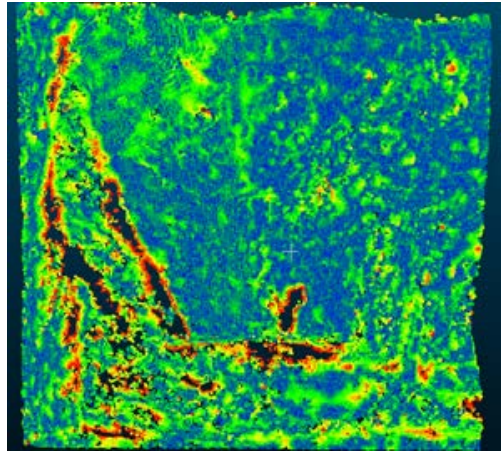

a)

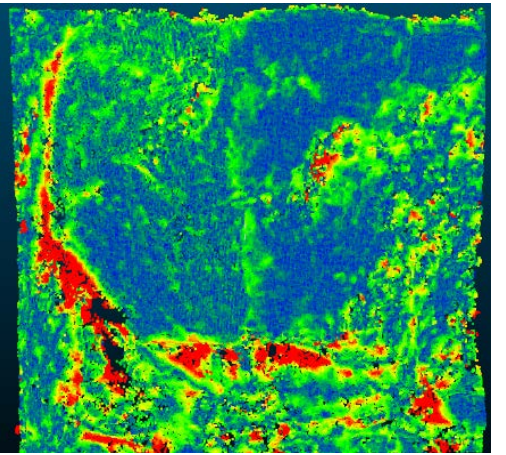

(b)

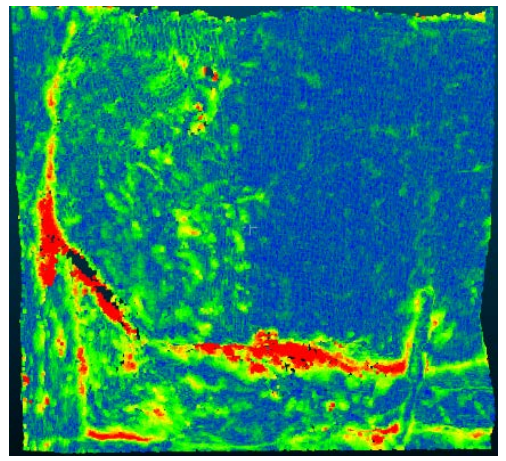

(c)

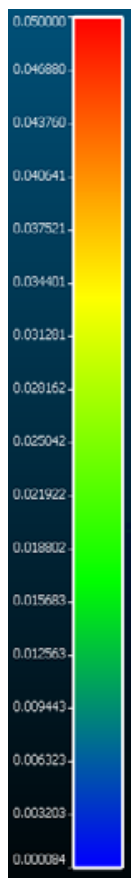

Figure 8. Detail of the same rock feature for the photogrammetric models acquired at different times indicated with dashed yellow circle in the Figure 7: on the top the RGB models, on the bottom the error scale maps (colour code as in Figure 7): a) Block T2.1 acquired at 11:00, b) Block T2.2 acquired at 12:00, c) Block T2.3 acquired at 13:00.

\section{CONCLUSIONS}

In this work, preliminary tests for the assessment of a new lowcost photogrammetry system are presented. Two different test sites were considered to calibrate the low-cost photogrammetric system and to assess its performance. The first test site (T1) was the facade of a building at the University of Newcastle. The second test site (T2) pictured a small rock wall at Pilkington Street Reserve. In the latter case, images were acquired at different times and using two different acquisition techniques: single image acquisition and average of three consecutive frames. In both tests sites, the image blocks were oriented in self calibration using GCPs.

The results obtained in this first series of tests conducted at T1 showed that the implemented calibration procedure is still an open issue for this low-cost sensors. On one hand, a precalibration strategy should grant a more reliable procedure, by implementing a properly configured image block geometry, and reduce correlations between parameters. On the other hand, the results showed that using a fixed set of calibration parameters can produce inaccurate results, mainly due to the unstable geometry of the optics and acquisition sensor. In this context, a selfcalibration procedure can significantly improve the final output. However, considering that just few (in the worst case just two) acquisition units are used (each one with its own calibration parameters) and probably just few (or none) ground control points are available to constrain the BBA, it is very likely that self-calibration could results in an ill-posed estimation of the IO parameters. More investigations are needed to confirm this. Control points were used for the evaluation of the accuracy of the bundle block adjustment. For both test sites, the root mean squares error differences on the check points revealed accuracies around $1 \mathrm{~cm}$ in the BBA. This is in the order of the GSD.
Digital models were also generated with the maximum level of details available in PhotoScan. Nevertheless, due to the texture of the building of $\mathrm{T} 1$, the uniform colour patterns, the presence of an opaque surface (on the left), and a uniform metal structure on the left and on the top, the reconstructed models are very noisy and not useful for a complete and accurate reconstruction of the object. On the contrary, the generated 3D models obtained from five different blocks in the second test site T2 look complete without evident noise. In addition, the comparisons with the TLS reference model indicate that the accuracy of the photogrammetric models of T2 is in the order of the GSD (0.8 $\mathrm{cm})$. Some systematic effects due to the IO parameters estimation are still present. A detailed analysis of the reconstructed models highlights the influence of the vegetation areas and of the orientation of the sun during the image acquisition, especially for such low-cost sensors. Indeed, the image block captured in better exposure conditions reveals greater uniformity and consistency with the TLS model.

At this stage, the comparisons between the two different acquisitions methods adopted in $\mathrm{T} 2$ indicates that averaging multiple images generally does not have significant advantages. In particular, weather conditions, such as strong wind, could affect the image acquisition with the averaging multiple images method introducing more noise. Nevertheless, the tests conducted at T2 revealed that the PiCamera Module V2 produces images that are suitable for the complete reconstruction of the digital models.

Additional tests will be performed for improving the quality of acquisition, using different set ups of the optic parameters (e.g. ISO, brightness, etc.). Improving the resolution and, hence reducing the GSD, is another challenge for the proposed photogrammetric system. More testing is currently underway with low-cost lenses of various focal lengths. 


\section{ACKNOWLEDGEMENTS}

The financial support of the Australian Research Council (LP160100370) provided to the Newcastle authors is greatly acknowledged.

\section{REFERENCES}

Agisoft Lens, 2017. Agisoft Lens User Manual, Version 1.3, http://downloads.agisoft.ru/lens/doc/en/lens.pdf.

Agisoft PhotoScan, 2017. Agisoft PhotoScan User Manual Professional Edition, Version 1.3, http://www.agisoft.com/pdf/photoscan_1_3_en.pdf.

Bahrudin, M.S.B., Kassim, R.A., and Buniyamin, N., 2013. Development of Fire Alarm System using Raspberry Pi and Arduino Uno. In: Electrical, Electronics and System Engineering (ICEESE), 2013 International Conference on, IEEE, pp. 43-48.

CloudCompare (version 2.9.beta) [GPL software] 2017. CloudCompare User Manual, Version 2.6.1, http://www.cloudcompare.org/doc/qCC/CloudCompare\%20v2.

6.1\%20-\%20User\%20manual.pdf.

Cooper, J., Azhar, M., Gee, T., Van Der Mark, W., Delmas, P., and Gimel'farb, G., 2017. A Raspberry Pi 2-based stereo camera depth meter. In: Machine Vision Applications (MVA), 2017 Fifteenth IAPR International Conference on. IEEE, pp. 274-277.

Cron, 2017. https://help.ubuntu.com/community/CronHowto (Accessed on July 27, 2017).

Duane, C. B. (1971). Close-range camera calibration. Photogramm. Eng, Vol. 37- 8, pp. 855-866.

Engel, P., and Schweimler, B., 2016. Development of AN OpenSource Automatic Deformation Monitoring System for Geodetical and Geotechnical Measurements. In: The International Archives of Photogrammetry, Remote Sensing and Spatial Information Sciences, Vol. XL-5/W8, pp. 25-30.

Ferdoush, S., and Li, X., 2014. Wireless sensor network system design using Raspberry $\mathrm{Pi}$ and Arduino for environmental monitoring applications. In: Procedia Computer Science, Vol. 34, pp. 103-110.

Kochláň, M., Hodoň, M., Čechovič, L., Kapitulík, J., and Jurečka, M., 2014. WSN for traffic monitoring using Raspberry Pi board. In: Computer Science and Information Systems (FedCSIS), 2014 Federated Conference on. IEEE, pp. 10231026.

Jindarat, S., and Wuttidittachotti, P., 2015. Smart farm monitoring using raspberry pi and arduino. In: Computer, Communications, and Control Technology (I4CT). IEEE, pp. 284-288.

Leccese, F., Cagnetti, M. and Trinca, D., 2014. A smart city application: A fully controlled street lighting isle based on Raspberry-Pi card, a ZigBee sensor network and WiMAX. In: Sensors, Vol. 14-12, pp. 24408-24424.

Muller, C.L., Chapman, L., Johnston, S., Kidd, C., Illingworth, S., Foody, G., Overeem, A. and Leigh, R.R., 2015. Crowdsourcing for climate and atmospheric sciences: current status and future potential. In: International Journal of Climatology, Vol. 35-11, pp. 3185-3203.
Neves, R., and Matos, A.C., 2013. Raspberry PI based stereo vision for small size ASVs. In: OCEANS - San Diego, pp. 1-6.

Nguyen, H.Q., Loan, T.T.K., Mao, B.D., and Huh, E.N., 2015. Low cost real-time system monitoring using Raspberry Pi. In: Ubiquitous and Future Networks (ICUFN), 2015 Seventh International Conference on, IEEE, pp. 857-85.

Nikhade, S.G., 2015. Wireless sensor network system using Raspberry $\mathrm{Pi}$ and zigbee for environmental monitoring applications. In: Smart Technologies and Management for Computing, Communication, Controls, Energy and Materials (ICSTM), 2015 International Conference on, IEEE, pp. 376-381.

Prasad, S., Mahalakshmi, P., Sunder, A.J.C., and Swathi, R., 2014. Smart Surveillance Monitoring System Using Raspberry PI and PIR Sensor. In: Int. J. Comput. Sci. Inf. Technol, Vol. 56, pp. 7107-7109.

$\begin{array}{lllll}\text { Raspberry } & \mathrm{Pi} & 2 & \text { Model } & \text { B, }\end{array}$ https://www.raspberrypi.org/products/raspberry-pi-2-model-b/ (Accessed on July 27, 2017).

Raspberry Pi Camera Module V2, 2017. https://www.raspberrypi.org/products/camera-module-v2/ (Accessed on July 27, 2017).

Roncella, R., and Forlani, G., 2015. A fixed terrestrial photogrammetric system for landslide monitoring. Modern Technologies for Landslide Monitoring and Prediction. Springer Berlin Heidelberg, pp. 43-67.

Schön, A., Streit-Juotsa, L., and Schumann-Bölsche, D., 2015. Raspberry Pi and Sensor Networking for African Health Supply Chains. In: Operations and Supply Chain Management, Vol. 83, pp. 137-145.

Zhuang, K., Yang, C., Yang, Y., Liu, J., and Jiang, N., 2016. Intelligent stereo camera mobile platform for indoor service robot research. In: Computer Supported Cooperative Work in Design (CSCWD), 2016 IEEE 20th International Conference on, IEEE, pp. 384-388. 